\title{
Homozygous Sickle Cell Anemia and Secondary Complications: A Case Study
}

\author{
CHRISTOPHER J YONTZ, KATHY V WALLER
}

\begin{abstract}
A 26-year-old African-American male presented with chest and back pain, fatigue and a history of the following: homozygous sickle cell anemia, pain crises, stroke, hip replacement following avascular necrosis of the femoral head, priapism, chronic transfusions, iron overload, hypertension, migraine headaches, port infections, depression and type II diabetes.
\end{abstract}

ABBREVIATIONS: ACS = acute chest syndrome; $\mathrm{AVN}=$ avascular necrosis; $\mathrm{CVA}=$ cerebrovascular accident; $\mathrm{MRI}=$ magnetic resonance imaging; $\mathrm{NTBI}=$ non-transferrin bound iron; $\mathrm{PHT}=$ pulmonary hypertension; $\mathrm{TCD}=$ transcranial Doppler ultrasonophraphy

INDEX TERMS: Red cell exchange transfusion, iron overload, sickle cell anemia, sickle cell crisis

Clin Lab Sci 2011;24(2):78

Christopher J. Yontz, The Ohio State University, Columbus, $\mathrm{OH}$

Kathy V. Waller, Ph.D., CLS, The Ohio State University, Columbus, $\mathrm{OH}$

Address for Correspondence: Christopher J. Yontz, The Ohio State University, 535 Atwell Hall, 453 W. $10^{\text {th }}$ Avenue, Columbus, $\mathrm{OH}$ 43210, 419-357-5031, yontz.17@buckeyemail.osu.edu

\section{CASE HISTORY}

\section{Patient history}

The patient is a 26-year-old African America male with a history of homozygous sickle cell anemia with multiple secondary complications. He was diagnosed in 1983 near the time of birth. His family history includes sickle cell disease in two cousins and sickle cell trait in both of his parents and one of his two brothers. The patient's second brother, other than being a type II diabetic, is considered healthy.

He was initially seen at the University hospital in the hematology clinic in 2000 as a 16-year-old with at least II-B and possibly III-A Avascular Necrosis (AVN) in his left hip. Avascular Necrosis is a gradual wearing of a weight-bearing surface, in this case the femoral head, which can lead to the collapse of the hip. ${ }^{1}$ The stages of necrosis range from stage I to stage IV, with stage IV being a completely flattened femoral head. This patient had progressed to stage III-B, meaning that his femoral head was aspherical and was beginning to show signs of collapse. Upon his first visit, the physician also learned of a past history of sickle cell disease, a Cerebrovascular Accident (CVA), or stroke around the age of seven, development of hypertension, migraine headaches, and chest fracture repair.

Complications secondary to sickle cell disease and occurring from 2000 to the time of admission in 2009 included AVN, sickle cell pain crises of approximately 25 times per year, musculoskeletal chest and back pain at rest and also during activity, night pain, as well as priapism. Other health complications included Type II diabetes, depression, and Tinea-like skin lesions on the upper left extremities and face.

Prior to hip replacement in 2002, the patient was noncompliant in using crutches and other therapy. He received a total left hip replacement due to AVN. He has also been receiving numerous units of red cells, typically in the form of red cell exchanges, since December of 2000. The transfusions are usually performed once a month; these have led to iron 


\section{CLINICAL PRACTICE}

overload. The patient has multiple port abscesses as well as port infections. He is in and out of the hospital frequently due to sickle cell pain crises.

The patient has been treated with a variety of medications for migraine headaches, pain, hypertension, iron overload, depression, and diabetes. Hydroxyurea, a cytoxic drug, was used in order to increase the percentage of hemoglobin $\mathrm{F}$ in an attempt to lessen his hemolytic and pain crises. However it was found that the patient was allergic to this agent. He was also allergic to penicillin, vancomycin and cephalosporins.

\section{Results from physical examination}

Over the years, the patient's physical examinations have generally been unremarkable, other than the musculoskeletal chest and back pain due to sickle cell pain crises. His physicians have noticed mild scleral icterus, Tinea-like lesions on the face and left, upper extremities, and occasional fatigue.

\section{Significant diagnostic laboratory tests}

Table 1 presents his laboratory values from 2002. Most notable are the data from hemoglobin $S$ quantitation and elevated ferritin levels. The patient's CBC in 2009 (Table 2) shows a normocytic, normochromic anemia. With red cell exchange for patients with sickle cell disease the concept is not to replace hemoglobin because the patient is "anemic" it is to replace the altered/abnormal hemoglobin (SS) with physiologically active hemoglobin (AA) in large amounts. Typically the patients end up at the hemoglobin they started with which is around 9 and no higher than 10. Throughout the years, his white blood cell counts and platelet counts have usually been increased. Hemoglobin evaluation by electrophoresis shows increased hemoglobin S, generally around $35 \%$ pre-transfusion and $10 \%$ post-transfusion. His hemoglobin solubility screen is consistently positive. The patient's current conditions require monthly monitoring of ferritin, due to iron overload that is being treated with Deferasirox. His liver and kidney function tests, although slightly elevated, are generally fairly stable. Overall, his bilirubin is generally between 3 and $4 \mathrm{mg} / \mathrm{dL}$, with his direct bilirubin being around $0.5 \mathrm{mg} / \mathrm{dL}$. The patient's LDH level is usually around 300 units/L and his creatinine about 0.35 $\mathrm{mg} / \mathrm{dL}$.
Table 1. Significant laboratory values from one of the patient's earlier visits on $08 / 21 / 2002$.

\begin{tabular}{lll}
\hline Analyte & Result & Reference Ranges \\
Red Blood Cell Count & $4.01 \times 10^{12} / \mathrm{L}$ & $4.30-5.60 \times 10^{12} / \mathrm{L}$ \\
Hemoglobin & $125 \mathrm{~g} / \mathrm{L}$ & $133-162 \mathrm{~g} / \mathrm{L}$ \\
Hematocrit & $0.365 \mathrm{~L} / \mathrm{L}$ & $0.388-0.464 \mathrm{~L} / \mathrm{L}$ \\
$\mathrm{MCHC}$ & $342 \mathrm{~g} / \mathrm{L}$ & $323-359 \mathrm{~g} / \mathrm{L}$ \\
$\mathrm{MCV}$ & $91.1 \mathrm{fL}$ & $79-93.3 \mathrm{fL}$ \\
Platelets & $412 \times 10^{9} / \mathrm{L}$ & $165-415 \times 10^{9} / \mathrm{L}$ \\
Hemoglobin A & $73.8 \%$ & $95-98 \%$ \\
Hemoglobin A2 & $3.0 \%$ & $1.5-3.1 \%$ \\
Hemoglobin S & $22.7 \%$ & Absent \\
Ferritin & $1650 \mathrm{ug} / \mathrm{L}$ & $29-248 \mathrm{ug} / \mathrm{L}$ \\
Hemoglobin Solubility & Positive & Negative \\
Screen & & \\
\hline
\end{tabular}

Table 2. Pre- and post-transfusion lab values from 10/09/2009 and $10 / 12 / 2009$, respectively. (9 Red cell units of type $\mathrm{B}+$, Kell negative blood were transfused on 10/12)

\begin{tabular}{llll}
\hline \multicolumn{2}{c}{ Pre-transfusion } & \multicolumn{2}{c}{ Post-transfusion } \\
Analyte & Result & Analyte & Result \\
Red Blood Cell & $3.39 \times 10^{12} / \mathrm{L}$ & Red Blood Cell & $3.50 \times 10^{12} / \mathrm{L}$ \\
Count & & Count & \\
Hemoglobin & $10^{9} \mathrm{~g} / \mathrm{L}$ & Hemoglobin & $10^{9} \mathrm{~g} / \mathrm{L}$ \\
Hematocrit & $0.32 \mathrm{~L} / \mathrm{L}$ & Hematocrit & $0.32 \mathrm{~L} / \mathrm{L}$ \\
MCHC & $332 \mathrm{~g} / \mathrm{L}$ & MCHC & $342 \mathrm{~g} / \mathrm{L}$ \\
MCV & $95.6 \mathrm{fL}$ & MCV & $91.6 \mathrm{fL}$ \\
Platelets & $475 \times 10^{9} / \mathrm{L}$ & Platelets & $153 \times 10^{9} / \mathrm{L}$ \\
Hemoglobin A & $57.9 \%$ & Hemoglobin A & $83.5 \%$ \\
Hemoglobin A2 & $3.02 \%$ & Hemoglobin A2 & $3.1 \%$ \\
Hemoglobin S & $35.9 \%$ & Hemoglobin S & $10.5 \%$ \\
Ferritin & Not done & Ferritin & $6142 \mathrm{ug} / \mathrm{L}$ \\
Hemoglobin & Positive & Hemoglobin & Positive \\
Solubility Screen & & Solubility Screen & \\
& & &
\end{tabular}

\section{Patient outcomes}

The patient benefited from an earlier total left hip replacement due to AVN, as well as stroke rehabilitation in his early years. To help deal with his numerous sickle cell crises, the patient was initially administered hydroxyurea, but he was found to have an allergy towards the drug. He is currently dealing with sickle cell disease with chronic exchange transfusions of Kell negative red cell units every four weeks instead of every six weeks, due to a long interval free of pain crises resulting from the more frequent red cell exchanges. Replacing the ports and allowing the infections to heal 


\section{CLINICAL PRACTICE}

with associated antibiotic therapy has been effective in treating the port infections.

In terms of the pain caused by the sickle cell pain crises, the patient is undergoing physical therapy and taking pain medications, which lower his daily resting pain level.

His immunizations are kept up to date to prevent further complications with his disease. The Tinea-like skin lesions have been cleared with medications and visits to his dermatologist.

The patient is evaluated by his family doctor every four to six weeks in order to monitor his ferritin levels and liver function tests due to the risk of iron overload from the constant red cell exchange transfusions. He is also receiving Deferasirox, an iron chelator, to ensure that secondary hemochromatosis does not occur.

\section{Quality of Life}

This patient, initially diagnosed at birth with sickle cell disease, has finished high school and is attempting to earn a degree in broadcasting and business. However, due to his chronic medical conditions, the patient has had to postpone his studies several times. He is single, unemployed, lives at home with his parents, and has an eight-year-old son. His conditions generally keep him from participating in many activities of everyday life, such as school and work, as he has been known to have as many as twenty-five pain crises per year.

When asked questions regarding depression, the patient states that he is depressed due to his conditions, as well as the death of one of his brothers. He was being treated synergistically for depression with both psychiatric counseling and medication; however he notes that no change had been seen with the use of medication and that he didn't care for his psychiatrist. He was encouraged to locate a new psychiatrist to continue his treatment of depression.

The patient's physicians have spoken with him about a possibly shortened life span due to his disease and complications. He understands the situation and has been educated on possibilities to prolong his life, such as a bone marrow transplant. A brother has been identified as a possible donor and he and his family are considering the options.

\section{EXPLANATION OF HOMOZYGOUS SICKLE CELL ANEMIA}

Homozygous sickle cell anemia is an autosomal codominantly inherited disorder that results in chronic hemolytic anemia. It is one of the most common genetic disorders in the world, seen most frequently in Sub-Saharan Africa, but also in geographic locations that are associated with the presence of malaria, or have immigrants from these locations. ${ }^{2}$ The genetic defect, a single point mutation resulting in the substitution of valine for glutamic acid at the 6 position on the beta globulin of chromosome 11, causes a sickling of hemoglobin in red blood cells. The sickled hemoglobin, referred to as Hemoglobin $S$, in the absence of oxygen, polymerizes, forming rods that develop the sickle or crescent shape of the red blood cell. (See Figure 1). Within the microcirculation, these cells stimulate a cascade of events that cause serious complications.

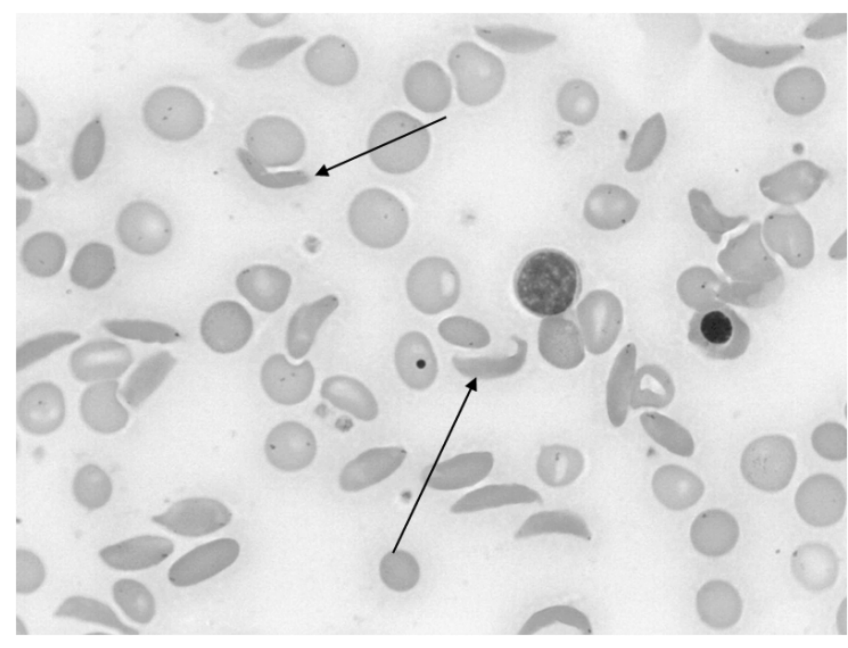

Figure 1. Peripheral blood smear from patient.

Arrows point to sickle cells. Note NRBC on left as well as HowellJolley Body and polychromasia.

The current median survival for sickle cell disease patients is 42 years for men, and 48 years for women, up from less than 20 years in the last three decades. ${ }^{1-3}$ The increase in survival rates for sickle cell patients has been due to increased screening in newborns, increased prophylaxis for the prevention of infections, advanced technology for detection and monitoring, and sustainable treatments for sickle cell anemia and its 


\section{CLINICAL PRACTICE}

secondary complications. ${ }^{2,3}$ Although an increase in life expectancy with sickle cell patients has been seen, this does not mean that the complications have diminished.

These patients live with a wide range of symptoms that vary in onset, severity, type and location. ${ }^{1}$ Due to the complexity of the variation in sequelae related to sickle cell disease, the management and treatment for the disease and accompanying conditions also vary from person to person. Individualized plans, including management of secondary conditions through various treatments and medications, allow the patient, physicians and scientists to determine possible strategies for dealing with this serious and long-term condition. ${ }^{1}$

\section{COMPLICATIONS OF SICKLE CELL ANEMIA}

The blockage of the small blood vessels by sickled cells causes episodes of vaso-occlusion, and pain crises, which characterize sickle cell disease clinically. ${ }^{1}$ However, sickle cell patients show a wide range of complications other than pain crises, including stroke, bacterial infection, sequestration of the spleen, avascular necrosis (AVN), leg ulcers, end-organ damage, renal disease, hypertension (HTN), acute chest syndrome (ACS), and priapism. ${ }^{2}$ As previously mentioned, these symptoms can come on all at once or gradually. They may occur frequently or sporadically, and can vary in type and location of pain. ${ }^{1}$

The patient introduced in this case study illustrates sickle cell anemia that includes several of the aforementioned complications. Although each individual case will present differently, complications are not all necessarily inevitable. The collection of secondary complications to sickle cell anemia that are unique to this patient, including stroke, pain crises, avascular necrosis of the left hip, hypertension, priapism, Tinea-like skin lesions on the upper extremities, and iron overload will be discussed next in order to gain greater understanding of the pathophysiology of sickle cell anemia.

At the age of seven, the patient experienced a cerebrovascular accident (CVA), or stroke, evidenced by multiple white matter lesions seen with Magnetic Resonance Imaging (MRI). Young children with sickle cell anemia are at very high risk for strokes because of the vascular occlusion and intravascular hemolysis seen with sickle cells. These abnormalities in the microvasculature eventually reach the brain, causing the child to have a stroke. Currently, abnormalities in the cerebral vasculature can be detected using Transcranial Doppler Ultrasonography (TCD) by measuring the velocity of blood flow in the brain. However this patient suffered a stroke 19 years prior to his current situation. It has been suggested that once a pediatric sickle cell patient has been considered at high risk for stroke, red cell transfusions can significantly reduce that risk when performed regularly. ${ }^{4}$

Sickle cell pain crises, which are responsible for the patient's back and chest pain, are known as the hallmarks of sickle cell anemia. ${ }^{2}$ These episodes of pain, such as acute chest syndrome, can be explained by a difficult mechanism resulting in red cell adherence to the vascular endothelium and activation of coagulation factors. When pain accompanies sickle cell anemia, tissue injury is being reflected. ${ }^{3}$ This patient has had a history of pain crises, occurring as many as 25 times per year and usually taking place two or three weeks posttransfusion. These episodes have led to as many as 50\% hospitalizations throughout a year. In fact, vasoocclusive pain is the most common reason for hospitalization among sickle cell patients. The pain itself is not life threatening, but secondary complications due to inadequate treatment can be fatal. Current studies are examining at ways to develop longterm management plans for pain crisis patients to recognize and manage their pain from home, without having to be admitted to the hospital. ${ }^{1}$

Hypertension and priapism can both be explained as manifestations of a hemolysis-related syndrome. Pulmonary hypertension (PHT) has emerged as a new complication for patients with various kinds of hemolytic anemia. The rationale behind this issue may be due to a high cardiac compensating output related to the anemia. Correction of the anemia by way of exchange transfusion, or administration of hydroxyurea in order to increase Hemoglobin F, may allow the patient to develop healthier red cells with normal function in relation to the vascular endothelium. In children with PHT, acute chest syndrome, asthma, and bacterial sepsis are common, while in adults, renal and 


\section{CLINICAL PRACTICE}

liver dysfunction can be seen. ${ }^{2}$ When the patient presented here was seen by a hematologist, he was already taking medication to lower his blood pressure. Priapism, which is the inability to return an erect and often painful penis to its normal state, found in 10 to $40 \%$ of adult males with sickle cell anemia, is also associated with hemolytic disorders. Its mechanism is not fully understood. ${ }^{2,3}$ This patient occasionally presented to his physician with priapism, which required surgical blood extraction from the penis at least once.

Avascular necrosis (AVN) occurs in sickle cell patients due to the increased intramedullary synthesis of blood cells of the bone marrow in an attempt to compensate for the hemolytic anemia. Necrosis typically occurs at the femoral head due to increasing pressure on the femoral head and a decrease in tenuous blood supply. A lack of blood supply to the bones, especially the femur, causes bone death to the weight-bearing surface of the head of the bone. ${ }^{3}$ At the age of 18 , this patient underwent a total left hip replacement because of AVN. Recent studies show that physical therapy can protect the hip from collapsing just as effectively as surgery if it is followed with persistence. Although AVN is certainly not a new finding with sickle cell patients, its prevalence is increasing. ${ }^{3}$

Tinea-like skin lesions were seen on the upper extremities and face of this patient on several occasions, in which case he was referred to a dermatologist for treatment. He also experienced several transfusion port infections, which required the removal of the port and reinstallation at a different site. The infections were treated with intravenous antibiotics. Sickle cell patients are generally susceptible to infections because the spleen in the first organ damaged by the sickle cells in the microcirculation. Bacteria such as Streptococcus pneumoniae are a common cause of bacteremia, while Salmonella sp. and Staphylococcus aureus are common causes of osteomyelitis in sickle cell patients. Infants are now screened at birth for sickle cell anemia not only for diagnostic purposes but also to prevent early death by bacterial sepsis. Children with sickle cell disease are kept up to date with immunizations and are treated with penicillin prophylaxis no later than three months of age. ${ }^{2}$ This type of prevention can be maintained throughout life. Unfortunately in this case, the patient was allergic to penicillin, vancomycin, and cephalosporin, so treatment for bacterial infections has been difficult.

In this case, an indirect secondary complication to sickle cell anemia has been iron overload. Due to chronic transfusions, (in order to reduce risk of stroke and to treat symptomatic anemia), priapism, hypertension, and chronic pain, macrophages are overloaded with iron in the form of non-transferrin bound iron (NTBI). As units of transfused blood increase, iron storage increases proportionately. In order to prevent iron toxicity in sickle cell patients, iron overload must be evaluated and managed as the major long-term problem with transfusing these patients. ${ }^{3,5}$ Physicians administered, to the patient in this case, two different types of drugs that act together to reduce iron levels, that will be discussed in the treatment section.

\section{CORRELATION OF LABORATORY TESTING}

A complete blood count (CBC) and bone marrow examination need to be run to evaluate a suspected person with sickle cell anemia initially. The CBC usually shows a normocytic, normochromic anemia in a patient with sickle cell anemia. The bone marrow examination will show erythroid hyperplasia, since the bone marrow attempts to compensate for the hemolytic anemia. ${ }^{6}$

Hemoglobin solubility tests are done when the clinical presentation, $\mathrm{CBC}$ and bone marrow examination imply a diagnosis of sickle cell anemia. If the patient has sickle cell anemia, the solubility test will be positive, meaning Hemoglobin $S$ is present in the individual. With this patient, the physician requested the hemoglobin solubility test, which gave a positive result.

Hemoglobin electrophoresis can be performed using both alkaline and acidic mediums. Performing electrophoresis on cellulose acetate at $\mathrm{pH} 8.4$ for a patient with sickle cell anemia will result in a band migrating in the Hemoglobin $S$ region on the gel. However, Hemoglobin D and Hemoglobin G also migrate with Hemoglobin $S$ on cellulose acetate, so electrophoresis should be performed on a second gel using citrate agar at $\mathrm{pH}$ 6.2. The acidic agar will 


\section{CLINICAL PRACTICE}

separate Hemoglobin $S$ from Hemoglobin D and Hemoglobin G, confirming the source of sickle cells in a patient. ${ }^{6}$ Electrophoresis on both cellulose acetate and citrate agar in this patient showed the presence of Hemoglobin $S$ in higher than normal proportions, confirming sickle cell anemia.

High-pressure liquid chromatography (HPLC) is a more recent method for determining the relative portion of specific hemoglobins found in whole blood, measured in percentages. Automated systems, such as The Variant ${ }^{\mathrm{TM}}$ II Hemoglobin Testing System, used in the laboratory where this patient was evaluated, combine sample preparation, separation, and determination into one instrument. The hemoglobins are measured according to their retention times from injection of the sample into the instrument to their peak concentrations. Each type of hemoglobin has its own characteristic retention time window that is used in the determination of hemoglobins present in a whole blood sample. ${ }^{7}$

\section{TREATMENT}

The administration of hydroxyurea, long-term transfusions, and bone marrow transplants are three of the primary treatments associated with sickle cell patients. Hydroxyurea, a pharmacological agent, is commonly used in adults with sickle cell anemia. It is the only medication that has been approved for the treatment of these patients. Hydroxyurea acts to increase the levels of fetal hemoglobin, or Hemoglobin $F$, in sickle cell patients. Hemoglobin F has a higher affinity for oxygen than does normal hemoglobin and especially Hemoglobin S. Increasing fetal hemoglobin increases total hemoglobin while decreasing hemolysis and vaso-occlusion. ${ }^{8}$ Hydroxyurea treatment was started with the patient in this case; however he was found to have an allergy towards the agent, so treatment was ceased.

Transfusions are used not only to increase the levels of normal hemoglobin in these patients, but also to reduce risk of stroke and to treat some of the symptoms involved in the anemia, such as fatigue. ${ }^{3}$ In this case, the patient has been transfused every four to six weeks in order to reduce his agonizing pain crises. However, chronic transfusions add a life-threatening complication to the mix, known as iron overload, often requiring iron chelation. Since iron overload is an important long-term complication in patients receiving chronic transfusions, maximizing iron chelation efficiency is a topic that has received much attention among medical scientists and pharmacologists. ${ }^{5}$ The FDA has approved two drugs to treat iron overload in transfusion patients. Deferoxamine, which requires continuous infusion, is used in combination with deferasirox, an oral iron chelator have recently emerged as highly effective drugs. ${ }^{3}$ In this particular case, the patient has been treated with both deferoxamine and deferasirox. The goal of iron chelation is to maintain an iron balance by matching the amount of the iron excretion through chelation and the amount of iron that is loaded during transfusion. Deferasirox is highly effective in maintaining these results because it allows continuous chelation over a 24 -hour time period by simply having the patient take a once-daily oral medication. ${ }^{5}$

While hydoxyurea and chronic transfusions are helpful in managing the symptoms of sickle cell patients, bone marrow transplantation may be the only current cure for sickle cell anemia. In order to free sickle cell patients from the numerous manifestations of hemolytic anemia, bone marrow transplant candidates must have a suitable donor, who is usually a primary family member. In order to find a suitable donor, HLA matching must be performed on family members and the patient in order to determine if the patient is a good candidate. In those patients who do not reject the graft, this procedure increases patient survival, free from usual clinical manifestations. However, only 14 to $18 \%$ of sickle cell patients are able to find a suitable donor. The procedure is often limited to younger patients with severe complications in order to reduce the risk of graft rejection. Currently, treatment developments are focused toward less intense procedures and more immunosuppression, which would be beneficial for older patients who generally would not be considered for transplant. ${ }^{3,9}$ The patient in this case was referred to a bone marrow transplant center and his brother has submitted a blood specimen for HLA typing. However, the family of the patient and his brother are reluctant to go ahead with the procedure. 


\section{CONCLUSION}

Patients with sickle cell anemia, such as the patient in this case, are diagnosed and treated by a team of health care professionals in order to establish a diagnosis and to manage the anemia and secondary conditions that can be difficult to control in everyday life. Since the average life expectancy of sickle cell patients has increased, current research regarding sickle cell anemia and its complications is focused on an aging population of those with sickle cell disease as well as their long-term management. ${ }^{2,3}$ Individualized management plans in terms of treating complications are anticipated to continue to show signs of increased effectiveness and efficiency. Home management of complications such as pain crises may be in the near future for these patients. ${ }^{1}$ An increase in newborn screening for sickle cell disease or trait, advanced technologies for detection, increased prophylaxis for prevention of infections in those diagnosed, and sustainable treatments contribute to an increase in life expectancy and perhaps quality of life of sickle cell patients. ${ }^{2,3}$

\section{REFERENCES}

1. Wright K; Adeosun O. Barriers to effective pain management in sickle cell disease. Br J Nurs. 2009;18:158-61.

2. Driscoll MC. Sickle cell disease. Pediatr Rev. 2007;28:259-68.

3. Hagar W, Vichinsky E. Advances in clinical research in sickle cell disease. Br J Haematol. 2008; 141:346-56.

4. Adams RJ. Big strokes in small persons. Arch Neurol. 2007;64:1567-74.

5. Porter JB. Concepts and goals in the management of transfusional iron overload. Am J Hematol. 2007;82(12 Suppl):1136-9.

6. McKenzie SB, Williams JL. Clinical Laboratory Hematology. $2^{\text {nd }}$ ed. New Jersey: Pearson; 2010.

7. LaRue H, Gewirtz A. Variant II Hemoglobin Testing System for Quantifying Normal and Abnormal Hemoglobins. OSUMC Department of Clinical Laboratories. 2009.

8. Strouse JJ, Lanzkron S, Beach MC et al. Hydroxyurea for sickle cell disease: a systematic review for efficacy and toxicity in children. Pediatrics. 2008;122:1332-42.

9. Bhatia M, Walters MC. Hematopoietic cell transplantation for thalassemia and sickle cell disease: past, present and future. Bone Marrow Transplant. 2008;41:109-17.

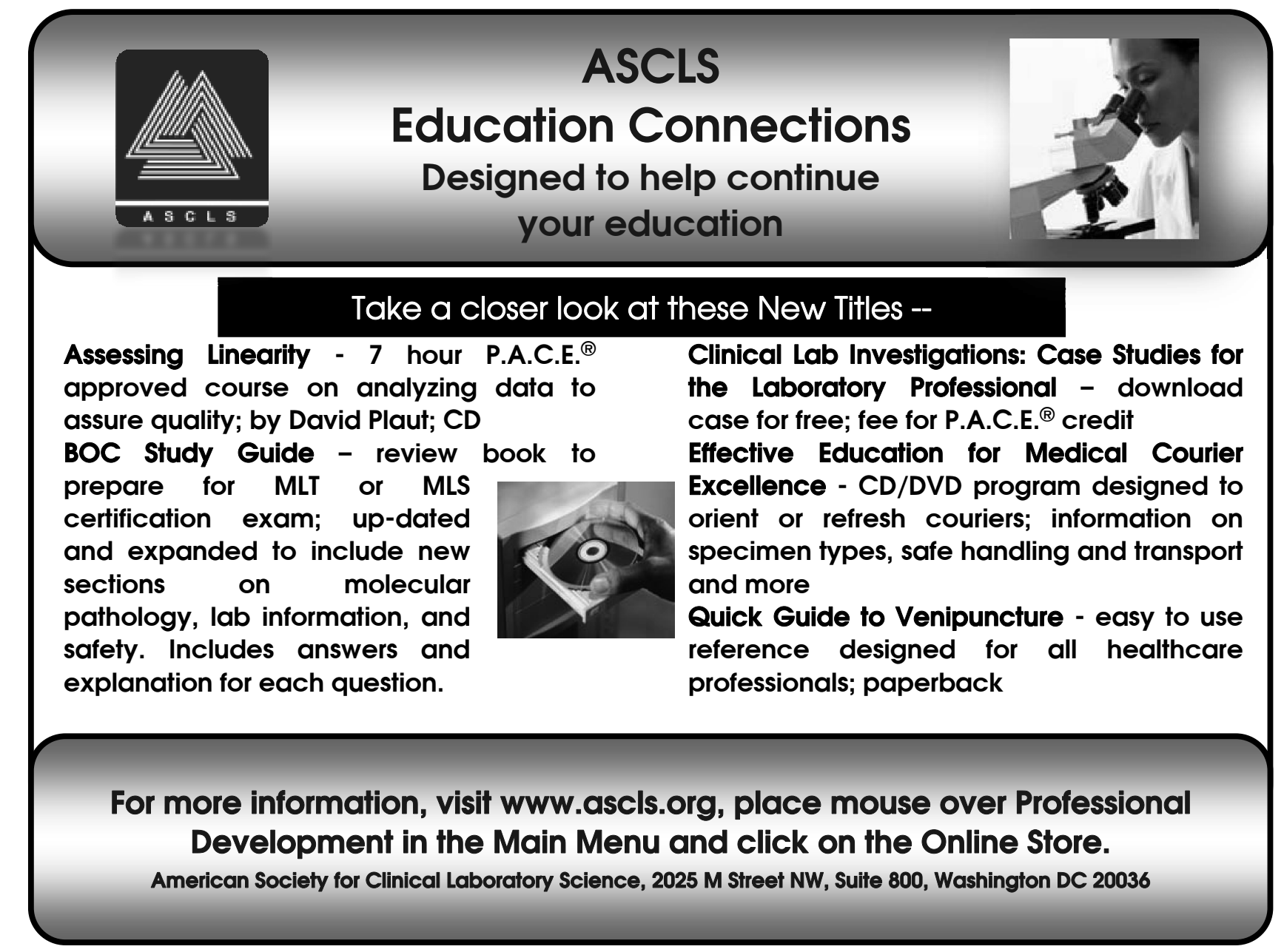

